\title{
Topological phases, topological flat bands, and topological excitations in a one-dimensional dimerized lattice with spin-orbit coupling
}

\author{
Zhongbo Yan and Shaolong Wan* \\ Institute for Theoretical Physics and Department of Modern Physics \\ University of Science and Technology of China, Hefei, 230026, P. R. China
}

(Dated: April 21, 2014)

\begin{abstract}
The Su-Schrieffer-Heeger (SSH) model describes a one-dimensional $Z_{2}$ topological insulator, which has two topological distinct phases corresponding to two different dimerizations. When spin-orbit coupling is introduced into the SSH model, we find the structure of the Bloch bands can be greatly changed, and most interestingly, a new topological phase with single zero-energy bound state which exhibits non-Abelian statistics at each end emerges, which suggests that a new topological invariant is needed to fully classify all phases. In a comparatively large range of parameters, we find that spin-orbit coupling induces completely flat band with nontrivial topology. For the case with non-uniform dimerizaton, we find that spin-orbit coupling changes the symmetrical structure of topological excitations known as solitons and antisolitons and when spin-orbit coupling is strong enough to induce a topological phase transition, the whole system is topologically nontrivial but with the disappearance of solitons and antisolitons, consequently, the system is a real topological insulator with well-protected end states.
\end{abstract}

PACS numbers: 71.10.Fd, 32.10.Fn, 03.65.Vf, 05.30.Rt

Introduction. - Since the discovery of integer quantum Hall effect [1], the concept of topology has become increasingly popular and important in condensed matter physics [2]. Topological phases, usually characterized by topological invariants which are connected to the energy spectrum and the nature of the wave function $[3,4]$, can appear in different dimensional systems with higher or lower symmetries $[5,6]$, and due to the robustness against disorder, they have the appealing potential application in topological quantum computation [7].

In the past few years, the theoretical predictions $[8,9]$ and the experimental observations [10, 11] of topological insulator have stimulated strong and continuous interest in predicting new classes of materials with nontrivial topological properties. From the lessons of topological insulators, we have learned that spin-orbit coupling is usually a natural ingredient to generate topological phases, e.g. topological superconductors which host desirable Majorana fermions [12, 13]. Inspired by the recent experimental realization of direct measurement of Zak phase in one-dimensional topological Bloch bands [14], and the realization of spin-orbit coupling in a onedimensional cold atomic system $[15,16]$, in this work we investigate the influence of spin-orbit coupling on the topology of Bloch bands in the one-dimensional doublewell optical lattice used in the experiment [14].

The one-dimensional double-well optical lattice is a cold atomic realization of the well-known Su-SchriefferHeeger (SSH) model [17]. The SSH model describes a $Z_{2}$ topological insulator, which has two topological distinct phases corresponding to two different dimerizations. In one dimension, Zak phase determines the topology of Bloch bands [18], and it takes value either $-\pi / 2$ or $\pi / 2$ (here we follow the choice of unit cell in Ref.[14], a different choice of unit cell gives the usual value 0 or $\pi$ ), and therefore, it is usually taken as the $Z_{2}$ invariant to characterize the two topological distinct phases, and a change of Zak phase indicates a topological phase transition. After introducing spin-orbit coupling, the extended SSH model still be a very simple model. However, spinorbit coupling will lift the original degeneracy of spin degree and induce strong influence on the structure of Bloch bands, and these consequently greatly rich the physics of the system. The main results induced by spin-orbit coupling include: (i) a series of topological phase transitions; (ii) the emergence of a new topological phase with single zero-energy bound state which exhibits non-Abelian statistics at each end; (iii) the formation of completely flat band with nontrivial topology; (iv) the change of the symmetric form of solitons and antisolitons; (v) the disappearance and the reappearance of solitons and antisolitons with the increase of the strength and the asymmetry of spin-orbit coupling.

Theoretical model. - We consider the one-dimensional double-well lattice realized in the experiment [14], but with the introduction of spin-orbit coupling. The Hamiltonian describing the system is given by

$$
\begin{aligned}
\hat{H}= & -\sum_{i, \sigma}\left(J \hat{a}_{i, \sigma}^{\dagger} \hat{b}_{i, \sigma}+J^{\prime} \hat{a}_{i, \sigma}^{\dagger} \hat{b}_{i-1, \sigma}+\text { h.c. }\right) \\
& +\sum_{i, \sigma}\left(\lambda \hat{a}_{i, \sigma}^{\dagger} \hat{b}_{i,-\sigma}-\lambda^{\prime} \hat{a}_{i, \sigma}^{\dagger} \hat{b}_{i-1,-\sigma}+\text { h.c. }\right),
\end{aligned}
$$

where $J, J^{\prime}$ denote modulated tunneling amplitudes within the unit cell, $\hat{a}_{i, \sigma}^{\dagger}\left(\hat{b}_{i, \sigma}^{\dagger}\right)$ are the particle creation operators for an atom with spin $\sigma(\uparrow$ or $\downarrow)$ in the $i$ th lattice cell. $\lambda, \lambda^{\prime}$ denote modulated spin-orbit coupling. For the special case, $\lambda=\lambda^{\prime}$, the spin-orbit coupling is just the form that can be realized in current cold-atomic experiments [19]. 
In the absence of spin-orbit coupling, i.e. $\lambda=\lambda^{\prime}=0$, the above Hamiltonian corresponds to the well-known Su-Schrieffer-Heeger model (SSH) of polyacetylene [17]. In the SSH model, spin degrees are decoupled and the Bloch bands for spin-up and spin-down are degenerate. For each spin degree, the topology of the Bloch bands is classified by the $Z_{2}$ invariant known as Zak phase. As the bands are degenerate, then if the lower band (the occupied band) is of nontrivial topology for one spin degree, the lower band for the other spin degree is also topologically nontrivial, this implies that the number of bound states for each end is even. Due to the degeneracy, the upper Bloch band and the lower Bloch band for spin-up and spin-down are simultaneously touched at the topological phase transition point $J=J^{\prime}$, as a result, the change of the Zak phase accompanying with the topological phase transition is $2 \pi$, instead of $\pi$.

After the appearance of spin-orbit coupling, the spin is no longer a good quantum number, and the degeneracy of the Bloch bands will be lifted, then by tuning the strength and asymmetry of the modulated spin-orbit coupling, only one of the lower band corresponding to one of the two helicities will undergo the topologically trivial to non-trivial or non-trivial to trivial transition, and the other lower band will keep its topology. This indicates that if the system is of nontrivial topology in the absence of spin-orbit coupling, then even the spin-orbit coupling drives a topological phase transition, the system is still topologically nontrivial, but with a change of the number of end bound states. This suggests that using a $Z_{2}$ invariant can no longer fully characterize all phases, and we need to introduce a new topological invariant. In the following, we will give a detail investigation of the influence of spin-orbit coupling.

The Hamiltonian (1) in momentum space takes the form,

$$
\begin{array}{r}
\hat{H}=\sum_{k} \hat{h}(k)=\sum_{k}\left\{-\left[\left(\operatorname{Re} \rho_{k}\right) \sigma_{x}-\left(\operatorname{Im} \rho_{k}\right) \sigma_{y}\right]\right. \\
\left.+\left[\left(\operatorname{Re} \delta_{k}\right) \sigma_{x}-\left(\operatorname{Im} \delta_{k}\right) \sigma_{y}\right] \tau_{x}\right\},
\end{array}
$$

where $\sigma_{x}, \sigma_{y}$ are the Pauli matrices for sublattice and $\tau_{x}$ is the Pauli matrix for spin, and

$$
\begin{aligned}
& \rho_{k}=J e^{i k d / 2}+J^{\prime} e^{-i k d / 2}=\left|\epsilon_{1}(k)\right| e^{i \theta_{1}(k)}, \\
& \delta_{k}=\lambda e^{i k d / 2}-\lambda^{\prime} e^{-i k d / 2}=\left|\epsilon_{2}(k)\right| e^{i \theta_{2}(k)} .
\end{aligned}
$$

The Hamiltonian obviously has particle-hole symmetry and chiral symmetry,

$$
\begin{aligned}
-\hat{h}(k) & =\sigma_{z} \hat{h}^{T}(-k) \sigma_{z}, \\
-\hat{h}(k) & =\sigma_{z} \hat{h}(k) \sigma_{z} .
\end{aligned}
$$

As $\sigma_{z} \sigma_{z}^{\dagger}=1$ and $\sigma_{z}^{T}=\sigma_{z}$, then according to Ref.[5], we know the Hamiltonian belongs to the symmetry class $B D I$ (chiral orthogonal), and the phases are classified by $Z$, an integer. In fact, the SSH model also belongs to this symmetry class, however, due to the degeneracy of the Bloch bands, a $Z_{2}$ invariant can fully classify the phases.

To determine the topology of the system, we need to determine the topology of the occupied Bloch bands, which can be realized by calculating the Zak phase of the corresponding band,

$$
\varphi_{Z a k}=i \sum_{\sigma} \int_{-G / 2}^{G / 2}\left(\alpha_{k, \sigma}^{*} \partial_{k} \alpha_{k, \sigma}+\beta_{k, \sigma}^{*} \partial_{k} \beta_{k, \sigma}\right) d k,
$$

where $\alpha_{k, \sigma}$ and $\beta_{k, \sigma}$ are the four components of a spinor $\mathbf{u}_{k}=\left(\alpha_{k, \uparrow}, \beta_{k, \uparrow}, \alpha_{k, \downarrow}, \beta_{k, \downarrow}\right)^{T}$, and $\mathbf{u}_{k}$ is the cellperiodical eigenstate of the corresponding band, satisfying $\hat{h}(k) \mathbf{u}_{k}=E_{k} \mathbf{u}_{k}$. Follow Ref.[20], we require that $\alpha_{k, \sigma}=\alpha_{k+G, \sigma}$ and $\beta_{k, \sigma}=-\beta_{k+G, \sigma}$, where $G=2 \pi / d$ is the reciprocal lattice vector.

From Eq.(2), we obtain the energy spectrums

$$
E_{k}= \pm \sqrt{\left|\rho_{k}\right|^{2}+\left|\delta_{k}\right|^{2} \pm \sqrt{2\left(\left|\rho_{k}\right|^{2}\left|\delta_{k}\right|^{2}+\operatorname{Re}\left(\rho_{k}^{2} \delta_{k}^{* 2}\right)\right)}} .
$$

We know the necessary condition for topological phase transition is the closure of the energy gap. When $\lambda=$ $\lambda^{\prime}=0$, the energy spectrums reduce to $E_{k}= \pm\left|\rho_{k}\right|$, it is direct to find that the energy gap is closed only when $J=J^{\prime}$ at $k=\pi / d$. In Ref.[14], it is shown that when $J>$ $J^{\prime}$, each of the two occupied bands has $\varphi_{Z a k}=\pi / 2$, and the system is topologically trivial, When $J<J^{\prime}$, each band has $\varphi_{Z a k}=-\pi / 2$, and the system is topologically nontrivial. To characterize the topology of Bloch bands conveniently, we define a new topological invariant $\nu$,

$$
(-1)^{\nu}=\operatorname{sgn}\left(\varphi_{Z a k}\right)
$$

$\nu=0$ corresponds to a trivial band and $\nu=1$ corresponds to a topologically nontrivial band. Based on this definition, the phases of the system are classified by an integer defined as $Z=\nu_{1}+\nu_{2}$, where $\nu_{1}$ is the topological invariant of the lower occupied band, and $\nu_{2}$ is the topological invariant of the upper occupied band. However, in the following we use $Z\left(\nu_{1}, \nu_{2}\right)$, instead of $Z$, to characterize the phases, because we can directly read the topology of every occupied band from $Z\left(\nu_{1}, \nu_{2}\right)$.

Topological phase transitions induced by spin-orbit coupling. - When $\lambda=\lambda^{\prime} \neq 0$, the degeneracy of the bands is lifted, and only the energy gap between the upper occupied band and the lower unoccupied band can be closed (see Fig.1(a)(b)(c)(d)). The condition for the closure of energy gap is now modified. The new criterion is given by

$$
\begin{aligned}
& \left|\epsilon_{1}\left(k_{c}\right)\right|=\left|\epsilon_{2}\left(k_{c}\right)\right|, \\
& \theta_{1}\left(k_{c}\right)-\theta_{2}\left(k_{c}\right)=0 \quad(\bmod \pi) .
\end{aligned}
$$

A little investigation shows that the two conditions can only be simultaneously satisfied when the parameters satisfy the relations $\left(\lambda^{2}+\lambda^{\prime 2}\right)-\left(J^{2}+J^{\prime 2}\right)= \pm 2\left(\lambda \lambda^{\prime}+J J^{\prime}\right)$. 

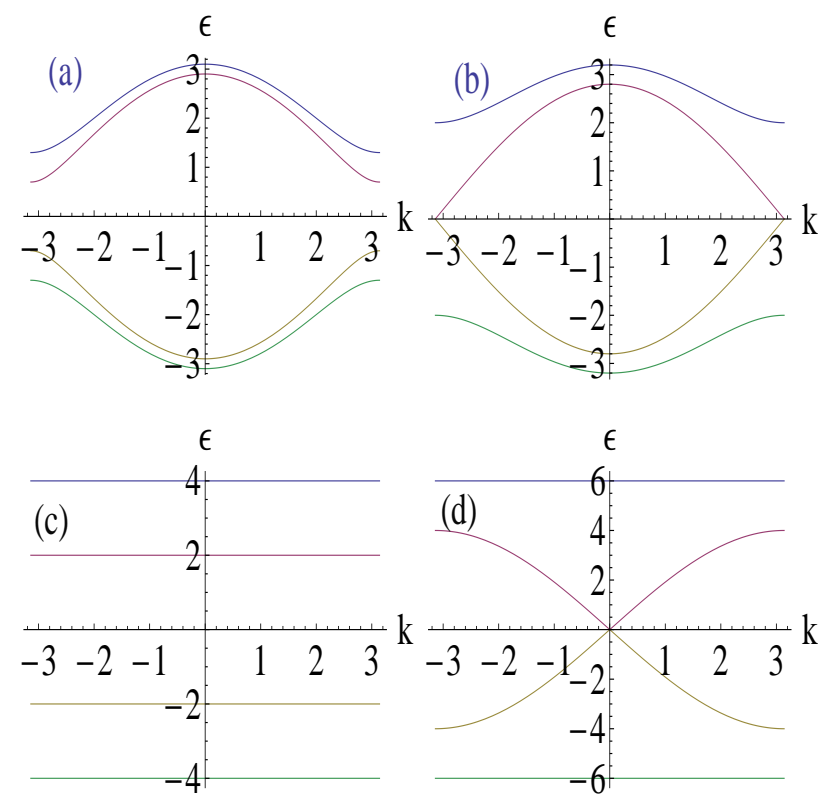

FIG. 1: (color online) Energy dispersion relation with parameters: (a) $J=2 J^{\prime}=1, \lambda=0.2, \lambda^{\prime}=0.1$. (b) $J=2$ $J^{\prime}=1, \lambda=0.6, \lambda^{\prime}=0.4$. (c) $J=2 J^{\prime}=1, \lambda=2, \lambda^{\prime}=1$. (d) $J=2 J^{\prime}=1, \lambda=4, \lambda^{\prime}=1$.

For weak spin-orbit coupling $\left(\lambda^{2}+\lambda^{\prime 2}<<J^{2}+J^{\prime 2}\right)$, the Bloch bands do not change much and the upper occupied band and the lower unoccupied band still touch at $k_{c}=\pi / d$ (see Fig.1(a)(b)). At the gap-closure point, the parameters should satisfy the relation $\left(\lambda^{2}+\lambda^{\prime 2}\right)-$ $\left(J^{2}+J^{\prime 2}\right)=-2\left(\lambda \lambda^{\prime}+J J^{\prime}\right)$. If we take $\lambda=\lambda^{\prime}=0$, this relation just gives $J=J^{\prime}$, agreeing with the result discussed above. From the criterion above, the parameter relation for the closure of energy gap is given by

$$
\left|J-J^{\prime}\right|=\lambda+\lambda^{\prime} .
$$

For the sake of discussion, here we first assume $J>J^{\prime}$. This assumption gives $J=J^{\prime}+\lambda+\lambda^{\prime}$. This simple relation indicates that spin-orbit coupling changes the transition point in a linear way.

For strong spin-orbit coupling $\left(\lambda^{2}+\lambda^{\prime 2}>J^{2}+J^{\prime 2}\right)$, the form of the Bloch bands has changed a lot and the upper occupied band and the lower unoccupied band now touch at $k_{c}=0$ (see Fig.1(c)(d)). The criterion for gap closure is $\left(\lambda^{2}+\lambda^{\prime 2}\right)-\left(J^{2}+J^{\prime 2}\right)=2\left(\lambda \lambda^{\prime}+J J^{\prime}\right)$. The parameter relation deduced from the criterion above is given by

$$
\left|\lambda-\lambda^{\prime}\right|=J+J^{\prime}
$$

To confirm whether the gap closure discussed above corresponds to a topological phase transition, we need to calculate the Zak phase of the upper occupied band before and after the closure of gap. We first consider the case with weak spin-orbit coupling. Before the discussion, we remind the fact that only when energy gap gets closed, the topology of the bands can change, and therefore, we can choose special value for the parameters in every gapped region to calculate the Zak phase. Before the closure of gap, based on Eq.(9), we choose $J=2$, $J^{\prime}=0, \lambda=1, \lambda^{\prime}=0$, a few steps of calculation shows that the four-component spinor $\mathbf{u}_{k}$ of the upper occupied Bloch band takes the form,

$$
\mathbf{u}_{k}=\frac{1}{2}\left(1, e^{-i k d / 2}, 1, e^{-i k d / 2}\right)^{T},
$$

then based on Eq.(5), it is direct to obtain $\varphi_{Z a k}(J>$ $\left.J_{c}\right)=\pi / 2\left(\right.$ where $\left.J_{c}=J^{\prime}+\lambda+\lambda^{\prime}\right)$, or equivalently, $\nu_{2}=0$. This indicates the system is a trivial insulator, agreeing with the fact that the parameters we choose above can be continued to the case with parameters $\lambda=$ $\lambda^{\prime}=0$ and $J>J^{\prime}$, without the closure of gap. After the closure of gap and the re-open of gap, based on Eq.(9), we choose Fig.1(c)'s parameters: $J=2, J^{\prime}=1, \lambda=2$, $\lambda^{\prime}=1$, then $\mathbf{u}_{k}$ of the upper occupied Bloch band is given by

$$
\mathbf{u}_{k}=\frac{1}{2}\left(1, e^{i k d / 2}, 1, e^{i k d / 2}\right)^{T},
$$

then we obtain $\varphi_{Z a k}\left(J<J_{c}\right)=-\pi / 2$, or equivalently, $\nu_{2}=1$. This indicates the upper occupied band which is completely flat (see Fig.1(c)) is of nontrivial topology. If we keep $J=\lambda$, we find that varying $\lambda^{\prime}$ with the constraint $\lambda^{\prime}<\lambda$ does not change the completely flat form of the upper occupied band. This suggests that the topological flat band can exist in a broad region. Flatbands are of great interest because they play an important role in the study of strong correlated phenomena. One-dimensional flat bands with nontrivial topology have already been considered in models similar to the SSH model (only similar in form, the underlying topology is different) and the authors have found the existence of fractional topological phase [21-23].

$\nu_{2}=1$ indicates when $\lambda+\lambda^{\prime}>J-J^{\prime}$, the system is driven into the topological phase $Z(0,1)$. As $J$ keeps larger than $J^{\prime}$, the dimerization of the topological phase $Z(0,1)$ is the same as the trivial phase $Z(0,0)$. This suggests an important fact that with the appearance of spin-orbit coupling, using the simple picture of dimerization to directly classify the phases is broken down.

Based on the analysis above, when $J=J_{c}$, accompanying the closure of energy gap, a topological phase transition takes place, with a change of Zak phase $\delta \varphi_{Z a k}=\pi$ $(\bmod 2 \pi)$.

With continuously increasing the strength of spin-orbit coupling, the re-open gap will get closed again. In the region $\left|\lambda-\lambda^{\prime}\right|>J+J^{\prime}$, we choose $J=1, J^{\prime}=0, \lambda=2$, $\lambda^{\prime}=0$ to calculate the Zak phase. The $\mathbf{u}_{k}$ of the upper 
occupied Bloch band is given by

$$
\mathbf{u}_{k}=\frac{1}{2}\left(-1, e^{-i k d / 2},-1, e^{-i k d / 2}\right)^{T},
$$

then the same procedure produces $\varphi_{Z a k}\left(\left|\lambda-\lambda^{\prime}\right|>J+\right.$ $\left.J^{\prime}\right)=\pi / 2$, or equivalently, $\nu_{2}=0$. The system return to the trivial phase $Z(0,0)$. Therefore, the two closures of gap both correspond to topological phase transition with a change of Zak phase $\delta \varphi_{Z a k}=\pi(\bmod 2 \pi)$.

Above, we have assumed $J>J^{\prime}$, if we instead assume $J^{\prime}>J$, we find the gap closure also always correspond to topological phase transition with a change of Zak phase $\delta \varphi_{Z a k}=\pi(\bmod 2 \pi)$. A big difference from the case with $J>J^{\prime}$ is that, for $J^{\prime}>J$, the topological phase transition is between two topological phases with different topological invariants, and therefore, the system always hosts end bound states. The only change is the number of the end bound states.

Based on the analysis above, the phase diagrams can be directly obtained. The phase diagrams for $J>J^{\prime}$ and $J<J^{\prime}$ have similar form. From Fig.2(a)((b)), we can see by continuously increasing the strength and asymmetry of spin-orbit coupling, the system first undergoes a topological phase transition from the trivial phase $Z(0,0)$ (topological phase $Z(1,1))$ to the topological phase $Z(0,1)(Z(1,0))$ and then undergoes another topological phase transition from the topological phase $Z(0,1)(Z(1,0))$ back to the trivial phase $Z(0,0)$ (topological phase $Z(1,1))$. Fig.2(c) shows that in the trivial phase $Z(0,0)$, there is no zero energy bound state at the end of the one-dimensional system. Fig.2(d)(f) show that the topological phase $Z(0,1)$ or $Z(1,0)$ hosts single zero-energy bound state at each end. Fig.2(e) shows that the topological phase $Z(1,1)$ hosts two zero-energy bound states at each end. The difference of the number of edge states suggests that the topological phase $Z(0,1)$ or $Z(1,0)$ is a new topological phase. As the Hamiltonian belongs to the symmetry class $B D I$, the single zeroenergy end state should be the same as the one found in Ref.[24] and therefore exhibits non-Abelian statistics. Consequently, the single zero-energy end state is similar to an unpaired Majorana fermion [25] and has the potential application in topological quantum computation.

The effect of spin-orbit coupling to topological excitations. - It is well-known that the most famous excitations in the SSH model are solitons and antisolitons which are movable [17]. They are the domain walls of the two topological distinct phases with different dimerizations. The physics of such domain walls is captured by the famous Jackiw-Rebbi model [26] and the TLM model [27]. Based on the Eq.(1), for weak spin-orbit coupling, the continuum Hamiltonian is given by

$$
\hat{h}(x)=-i v \partial_{x} \sigma_{z}+\Delta(x) \sigma_{x}+\left[i v^{\prime} \partial_{x} \sigma_{z}-\Delta^{\prime}(x) \sigma_{x}\right] \tau_{x},(14)
$$

where $v=\left(J+J^{\prime}\right) d / 2, v^{\prime}=\left(\lambda-\lambda^{\prime}\right) d / 2$, and $\Delta(x)=$ $J-J^{\prime}, \Delta^{\prime}(x)=\lambda+\lambda^{\prime}$ in the case of uniform dimerization.
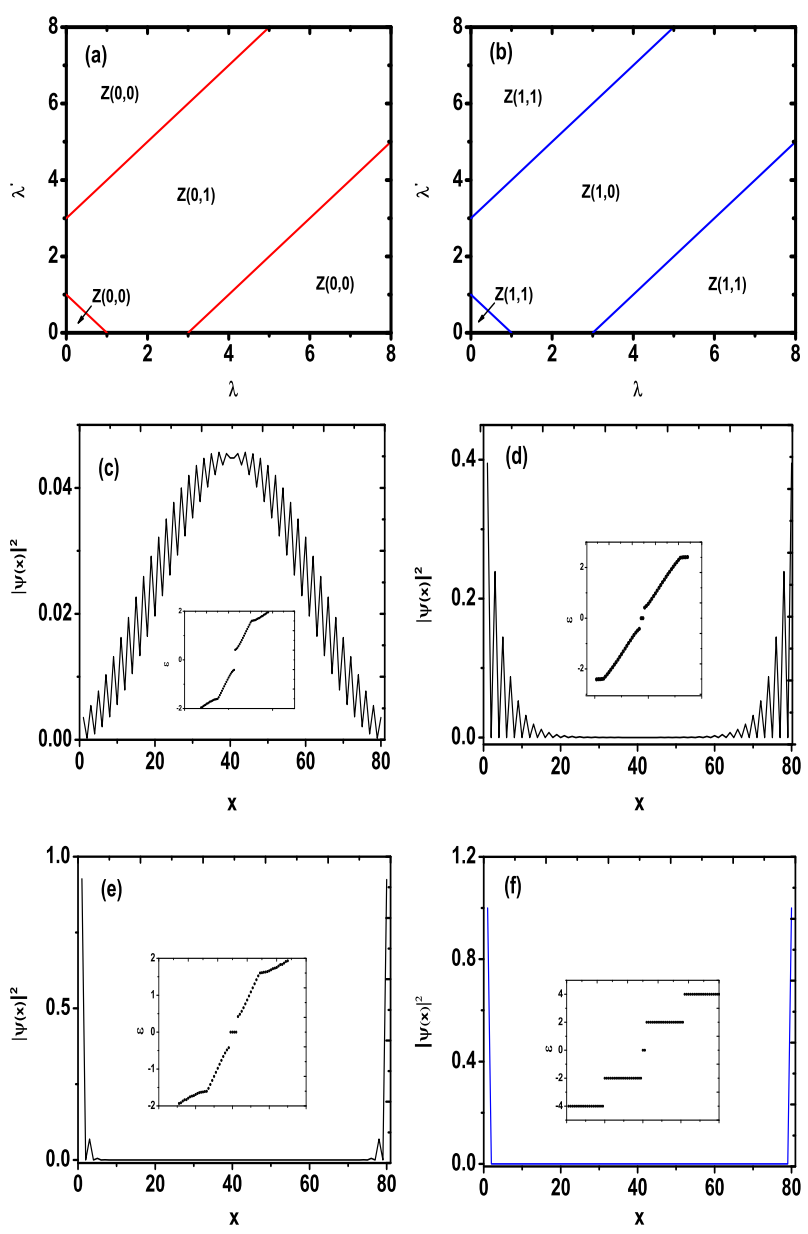

FIG. 2: (color online) (a)(b) are Phase diagrams, with parameters: (a) $J=2 J^{\prime}=1$. (b) $J=1 J^{\prime}=2$. (c)(d)(e)(f) show the wave functions for excitations with the lowest energy with parameters, (c) $J=2, J^{\prime}=1, \lambda=0.4, \lambda^{\prime}=0.2$, (d) $J=2, J^{\prime}=1, \lambda=0.6, \lambda^{\prime}=0.8$. (e) $J=1, J^{\prime}=2$, $\lambda=0.4, \lambda^{\prime}=0.2$. (f) $J=1, J^{\prime}=2, \lambda=1, \lambda^{\prime}=2$. The insets in $(\mathrm{c})(\mathrm{d})(\mathrm{e})(\mathrm{f})$ are the corresponding energy spectrums with $0,2,4,2$ in-gap zero-energy bound states, respectively.

By making a rotation of spin, $\tilde{h}(x)=e^{i \frac{\pi}{4} \tau_{y}} \hat{h}(x) e^{-i \frac{\pi}{4} \tau_{y}}$, we obtain

$$
\tilde{h}(x)=-i v \partial_{x} \sigma_{z}+\Delta(x) \sigma_{x}+\left[i v^{\prime} \partial_{x} \sigma_{z}-\Delta^{\prime}(x) \sigma_{x}\right] \tau_{z}
$$

the Hamiltonian now can be decoupled to two parts corresponding to two different helicities,

$$
\begin{gathered}
\tilde{h}_{+}(x)=-i\left(v+v^{\prime}\right) \partial_{x} \sigma_{z}+\left(\Delta(x)+\Delta^{\prime}(x)\right) \sigma_{x}, \\
\tilde{h}_{-}(x)=-i\left(v-v^{\prime}\right) \partial_{x} \sigma_{z}+\left(\Delta(x)-\Delta^{\prime}(x)\right) \sigma_{x} .
\end{gathered}
$$

For $J>J^{\prime}, \tilde{h}_{+}(x)$ always describes a trivial phase, and $\tilde{h}_{-}(x)$ may describe a trivial phase or a topological phase depending on the sign of $\left(\Delta(x)-\Delta^{\prime}(x)\right)$. For $J<J^{\prime}$, on the contrary, $\tilde{h}_{-}(x)$ always describe a topological phase, and $\tilde{h}_{+}(x)$ may describe a trivial phase or a topological phase depending on the sign of $\left(\Delta(x)+\Delta^{\prime}(x)\right)$. Based on 
the fact that the system undergoes a topological phase transition when $\Delta(x)-\Delta^{\prime}(x)=0$ for $J>J^{\prime}$ and $\Delta(x)+\Delta^{\prime}(x)=0$ for $J<J^{\prime}$, the parameter relation for uniform relation in Eq.(9) is reobtained. For strong spin-orbit coupling, as the energy bands now touch at $k_{c}=0$, therefore, the roles played by the kinetic term $-i v \partial_{x} \sigma_{z}$ and the order parameter term $\Delta(x) \sigma_{x}$ are exchanged. The discussion of the case with strong spinorbit coupling is straightforward, and we neglect it here.

For non-uniform dimerization, $\tilde{h}_{+}(x)$ describes topological excitations with one of the helicities labeled as "+", and $\tilde{h}_{-}(x)$ describes topological excitations with the other one labeled as "-". Based on Eq.(16), the decay properties of the wave functions take the form

$$
\varphi_{ \pm}(x) \propto \exp \left(-\frac{\left|\Delta(x) \pm \Delta^{\prime}(x)\right||x|}{\tilde{v}}\right),
$$

here we have used the assumption $\Delta(x)=-\Delta(-x)=$ $J-J^{\prime}>0$ for $x<0, \Delta^{\prime}(x)=\lambda+\lambda^{\prime}<J-J^{\prime}$ and Dirichlet boundary condition for simplicity, and $\tilde{v}=v \pm v^{\prime}$ for $\tilde{h}_{ \pm}(x)$, respectively. From Eq.(17), it is found that when $\Delta^{\prime}(x)=0$, the topological excitations have a symmetric structure, however, once $\Delta^{\prime}(x) \neq 0$, topological excitations with helicity "+" ("-") become more localized at the left (right) side and more extended at the right (left) side of the domain.

Another important result induced by spin-orbit coupling is that by increasing spin-orbit coupling to make $\lambda+\lambda^{\prime}>\left|J-J^{\prime}\right|$ (but $\left|\lambda-\lambda^{\prime}\right|<J+J^{\prime}$ ), although the dimerization configuration keeps the same, the topological properties of both sides are changed (as shown in Fig.2(a)(b)). A direct result of the simultaneous change is the disappearance of the moving topological excitations. It is direct to confirm this by noting that in this parameter region, the sign of $\left(\Delta(x)+\Delta^{\prime}(x)\right)$ is always positive and the sign of $\left(\Delta(x)-\Delta^{\prime}(x)\right)$ is always negative. Unlike the SSH model where the existence of moving topological excitations makes the system actually conducting and the end states in fact unprotected, the disappearance of the moving topological excitations indicates the system turns to be a real insulator in bulk, and consequently, the system is a real topological insulator with well-protected end states. The disappearance of the moving topological excitations also suggest the two topological phases denoted by $Z(0,1)$ and $Z(1,0)$ are the same, agreeing with the fact that the Hamiltonian is classified by an integer. Base on Fig.2(a)(b), topological excitations will reappear by further increasing the strength and the asymmetry of spin-orbit coupling.

Conclusion. - The introduction of spin-orbit coupling greatly riches the physics of SSH model. First, with the lift of the degeneracy, we find the usual $Z_{2}$ invariant classifying the phases of SSH model can no longer fully classify all phases. The new topological phase corresponding to $Z=1$ hosts interesting single zero-energy bound state which exhibits non-Abelian statistics at each end and is stable against the variation of dimerization, therefore, it can have great potential application in topological quantum computation. Second, in the region of topological phase $Z=1$, completely flat band with nontrivial topology can be formed by tuning the spin-orbit coupling. Third, for the case with non-uniform dimerization, spin-orbit coupling in weak region changes the symmetrical form of topological excitations, and with increasing the strength and the asymmetry of spin-orbit coupling, the topological excitations will disappear when a topological phase transition takes place and then reappear when another topological phase transition takes place. These results suggest spin-orbit coupling can be used to control the phases and the topological excitations of the system. The progress on experiments makes the observation of the new topological phase and the topological phase transitions induced by spin-orbit coupling in experiments within current ability.

Acknowledgments. - We would like to thank Xiaosen Yang for helpful discussions. This work is supported by NSFC. Grant No.11275180.

* slwan@ustc.edu.cn

[1] K. Klitzing, G.Dorda, M. Pepper, Phys. Rev. Lett. 45, 494 (1980).

[2] D. Thouless, M. Kohmoto, M. Nightingale, M. den Nijs, Phys. Rev. Lett. 49, 405, (1982)

[3] X.-G. Wen, Int. J. Mod. Phys. B 4, 2391990.

[4] X.-G. Wen, Int. J. Mod. Phys. B 6, 17111992.

[5] A.P. Schnyder et al, Phys. Rev. B 78, 195125 (2008).

[6] A. Y. Kitaev, AIP Conf. Proc. 1134, 22-30 (2009).

[7] A. Y. Kitaev, Ann. Phys. (N.Y.) 303, 2 (2003).

[8] C. L. Kane, Phys. Rev. Lett. 95, 1468022005

[9] B. A. Bernevig, T. L. Hughes, and S.-C. Zhang, Science 314, 1757 (2006).

[10] M. König et al, Science 318, 766 (2007).

[11] D. Hsieh, Nature 452, 970 (2008).

[12] Roman M. Lutchyn, Jay D. Sau, and S. Das Sarma, Phys. Rev. Lett. 105, 077001 (2010).

[13] J. Alicea, Phys. Rev. B 81, 125318 (2010).

14] M. Atala et al, Nat. Phys 9, 795 (2013).

[15] Y.-J. Lin, K. Jimenez-Garcia, and I. B. Spielman, Nature 471, 83 (2011).

[16] P. J. Wang et al, Phys. Rev. Lett. 109, 095301 (2012).

[17] W. P. Su, J. R. Schrieffer and A. J. Heeger, Phys. Rev. Lett. 42, 1698 (1979).

[18] J. Zak, Phys. Rev. Lett. 62, 2747 (1989).

[19] V. Galitski and I. B. Spielman, Nature 494, 49 (2013).

[20] M. J. Rice and E. J. Mele, Phys. Rev. Lett. 49, 1455 (1982).

[21] H. Guo and S. Q. Shen, Phys. Rev. B 84, 195107 (2011).

[22] H. Guo, S. Q. Shen, and S. Feng, Phys. Rev. B 86, 085124 (2012).

[23] J. C. Budich and E. Ardonne, Phys. Rev. B 88, 035139 (2013).

[24] J. Klinovaja and D. Loss, Phys. Rev. Lett. 110, 126402 (2013). 
[25] A. Y. Kitaev, Phys.-Usp. 44, 131 (2001).

[26] R. Jackiw, C. Rebbi, Phys. Rev. D 13, 3398 (1976).

[27] H. Takayama, Y. R. Lin-Liu, and K. Maki, Phys, Rev. B

21, 2388 (1980).

. 